\title{
Diagnostic and therapeutic approaches to multiple myeloma patients: 'Real-world' data from representative multicentre treatment surveys in Germany between 2008 and 2011
}

\author{
THOMAS M. MOEHLER ${ }^{1,5}$, MAXIMILIAN MERZ ${ }^{1}$, LENKA KELLERMANN ${ }^{2}$, \\ HARTMUT GOLDSCHMIDT $^{1,3}$ and WOLFGANG KNAUF ${ }^{4}$
}

${ }^{1}$ Department of Medicine V, University of Heidelberg, Heidelberg 69120; ${ }^{2}$ Oncology Information Service, Freiburg 79098 ;
${ }^{3}$ National Center for Tumor Diseases, Heidelberg $69120 ;{ }^{4}$ Onkologische Gemeinschaftspraxis, Frankfurt 60389 , Germany

Received May 26, 2015; Accepted April 29, 2016

DOI: $10.3892 / \mathrm{ol} .2016 .5375$

\begin{abstract}
A survey was conducted to investigate the standard of care for multiple myeloma in Germany, in order to clarify the status of implementation of international and national treatment guidelines. In addition, the changes in disease management over time were investigated by comparison with surveys conducted in 2008 and 2009. The survey captured a representative sample of 478 myeloma patients with a mean age of 67.9 years across various stages of the disease. Diagnostic approaches, prognostic aspects and treatment decisions were evaluated based on a survey conducted in 2011 in 58 representative centres in Germany, including university and non-university hospitals and office-based haematologists. Data were collected from chart reviews and were analysed retrospectively. Over time, an increasing number of patients were investigated for cytogenetic abnormalities (53\%). Age $<69$ years and lack of comorbid conditions were major determinants for cytogenetic testing. Bortezomib/chemotherapy-based regimens have become the preferred first-line treatments independent of planning autologous blood stem cell transplantation (ASCT) in first-line therapy. Thalidomide- and lenalidomide-based combination therapies are typically used as second-line treatments in $31 \%$ of patients. Compared with previous reviews, the frequency of ASCT was stable, at $~ 30 \%$ of patients. Younger age and indicators of more severe disease, such as the presence of CRAB criteria, influenced the decision in favour of performing ASCT. Compared to previous surveys, the requirement for erythropoietin and granulocyte colony-stimulating factor, as well as transfusions of red blood
\end{abstract}

Correspondence to: Professor Wolfgang Knauf, Onkologische Gemeinschaftspraxis, Im Prüfling 17-19, Frankfurt 60389, Germany E-mail: wolfgang.knauf@telemed.de

Present address: ${ }^{5}$ inVentiv Health Clinical, 233 Frankfurter Street, Neu-Isenburg 63263, Germany

Key words: multiple myeloma, novel agents, autologous stem cell transplantation cells and platelets, respectively, have decreased considerably. In summary, novel agents have led to a substantial change in the first-line and relapsed treatment approaches. Age and comorbidities remain major factors influencing treatment decisions, but cytogenetic testing to investigate myeloma-related risk profiles is increasingly integrated. The use of novel agents has affected supportive care, with reduced necessity for substitute blood products and reduced administration of bone marrow-stimulating factors.

\section{Introduction}

Treatment of symptomatic multiple myeloma has significantly improved progression-free and overall survival times over the past 20 years, with the introduction of autologous blood stem cell transplantation (ASCT) in the early 1990s and subsequently the approval of proteasome-inhibitors and immunomodulatory drugs (IMiDs) (1-3).

The 5-year overall survival rate has increased from $35 \%$ for the years of 1999-2001, to 44\% for the years of 2006-2009. In particular, the survival of patients aged $<50$ years has significantly improved, with 10 -year survival rates increasing from 25 to $41 \%(4,5)$.

These improvements in survival are not based on a single agent or single treatment strategy, but rather on the selection of sequential regimens and design of combination therapies. Prognostic tests have been developed simultaneously with the integration of novel agents into the treatment algorithms. In particular, molecular markers have been identified that may support treatment decisions (6).

The optimal integration of novel agents and the future development of effective combination therapies is a major goal in myeloma research. Several sets of guidelines have been published and revised over time that outline recommendations for first-line therapy, but also for relapsed and refractory patients $(2,3,7,8)$. Importantly, current treatment recommendations and guidelines integrate prognostic factors into the treatment decisions (7). Nonetheless, myeloma treatment still requires additional improvement, as many patients suffer from treatment-related complications and ultimately will succumb to disease progression. 
The present survey was conducted with the goal of investigating whether recent international treatment guidelines were applied to the routine diagnostic work-up of myeloma patients and were used for treatment decisions. The matter of how the diagnostic and treatment decisions have changed over time was also examined by comparison with previously published surveys (9).

The current study sought to provide information on real-world treatment and diagnostic decisions to support information on which regimen and treatment pathways are currently favoured by clinicians, and which regimen could be the basis for the future development of myeloma care.

\section{Patients and methods}

Study design. The goal of the current study was to analyse the pattern of multiple myeloma management in clinical practice. The analysis was based on an epidemiological database (TherapyMonitor compiled by OncologyInformationService, Freiburg, Germany), comprising 478 patients from 58 centres in Germany, namely 10 university hospitals, 27 community hospitals, and 21 office-based haematologists, with a treatment decision made for multiple myeloma in the 1st-2nd quarter of 2011. This constitutes a representative statistical sample regarding the distribution of treated patients with multiple myeloma ('treated prevalence').

Selection of the centres was based on a two-step procedure. A total of 817 centres involved in the treatment of multiple myeloma, including university hospitals $(n=36)$, community hospitals $(n=355)$ and office-based haematologists $(n=426)$, were contacted, and $15 \%$ of centres responded to provide their number of multiple myeloma patients (corresponding to $13 \%$ of the expected national prevalence). Based on this information, the distribution of multiple myeloma patients across the three main care providers was determined as follows: university hospitals, $20 \%$; community hospitals, 40\%; and office-based haematologists, 40\%. The total patient sample $(n=500)$ eligible for analysis after the quality check $(n=478)$ was defined by taking into account the treated prevalence per type of institution, the regional population density and health care structure. Reporting centres were selected randomly among the responders according to the date of response to the mailing. In the second step, demographic and clinical data were collected retrospectively in an online case report based on data in the clinical files. Patients who met the following criteria were included: Therapeutic decision (start, change, or end of therapy) between January and June 2011. Data was verified by central monitoring.

Demographics and clinical data. Patients' baseline characteristics [demographics, medical history, including comorbidity status, and multiple myeloma stage at initial diagnosis according to Durie-Salmon staging (10)] and clinical data were retrospectively extracted from chart reviews. All variables were reported by the treating physician and pseudonymised according to the available information in the patient chart. The online and external monitoring systems assessed the plausibility and completeness of the data. To descriptively assess the diagnostic, prognostic and treatment patterns according to age, information on cytogenetic testing (yes/no, results), and treatment (including its objective and reasons for starting therapy) was analysed for all patients. Patient characteristics are displayed in Table I.

Statistical analysis. Data presented in this article were analysed using descriptive and explorative methods. Diagnostic and treatment patterns were reported as absolute and relative frequencies.

For exploratory analyses, subgroup comparisons as well as bivariate analysis were performed. Differences in treatment patterns between subgroups and predictive factors were analysed using $\chi^{2}$ and Fisher's exact tests. All P-values are given as two-sided values, and $\mathrm{P}<0.05$ was considered to indicate a statistically significant difference. SPSS software, version 20 (IBM SPSS, Armonk, NY, USA) was used for analysis.

\section{Results}

Baseline diagnostics, prognostic laboratory tests and patient characteristics. The patient data reflected a representative multiple myeloma population, with a mean age of 67.9 years and a balanced distribution across the participating institutions. Patient characteristics are summarised in Table I.

Leading symptoms (largely unchanged over time) at diagnosis were non-specific (see summary in Fig. 1), as expected: Fatigue (47\%), bone pain (39\%), weakness (40\%) and decreased performance $(41 \%)$ were the most common.

The majority of patients $(79 \%)$ were in Durie-Salmon stage II and III at time of initial diagnosis. The concomitant disease spectrum was headed by renal insufficiency $(23 \%)$ and arterial hypertension (36\%). Importantly, the percentage of preexisting polyneuropathy was $5 \%$, and an additional $5 \%$ of patients had a psychiatric diagnosis, predominantly depression. The percentage of patients with malignant disease in their medical history has decreased over time, from $15 \%$ (2008), to $8 \%$ (2009), to $5 \%$ (2011).

A trend for a lower burden of disease at first diagnosis was observed, as $21 \%$ of patients were in Durie-Salmon stage I/II in 2011, whereas only $11 \%$ and $17 \%$ were in Durie-Salmon stages I/II in 2009 and 2008, respectively. Notably, the percentage of patients requiring systemic treatment in Durie-Salmon stage I/II did not increase, but rather decreased (Fig. 2A and B).

The acceptance of the International Staging System (ISS) (11) as a key prognostic factor was confirmed, as $75 \%$ of patients received a classification according to ISS in the current study, whereas $<20 \%$ had an ISS classification in the 2009 survey.

Compared with the previous survey, the acceptance of cytogenetic testing has increased from $23 \%$ in 2008 (9) and $35 \%$ in 2009 , to $53 \%$ in the current analysis across the institutional segments.

The decision tree method was used to identify the factors with major impacts on the choice of cytogenetic testing at initial diagnosis of multiple myeloma. In the group of patients without $\beta_{2}$-microglobulin $\left(\beta_{2}-\mathrm{MG}\right)$ assessment and aged $>69$ years, only $16 \%$ of patients underwent cytogenetic testing. By contrast, $94 \%$ patients in stage II without a concomitant disease received cytogenetic testing if a $\beta_{2}-\mathrm{MG}$ assessment was performed. In summary, $\beta_{2}-\mathrm{MG}$ and cytogenetic tests were often performed simultaneously or not performed at all. 
Table I. Patient characteristics.

\begin{tabular}{|c|c|c|c|}
\hline Characteristics (at initial diagnosis) $^{\mathrm{a}}$ & $\begin{array}{c}2011 \\
(n=478)\end{array}$ & $\begin{array}{c}2009 \\
(n=275)\end{array}$ & $\begin{array}{c}2008 \\
(n=386)\end{array}$ \\
\hline Age (mean in years) & 67.9 & 67.6 & 66.4 \\
\hline Gender ( $\%$ female $)$ & 43 & 49 & 41 \\
\hline Malignant disease in medical history (\%) & 6 & 8 & 15 \\
\hline \multicolumn{4}{|l|}{ Durie-Salmon stage $(\%)$} \\
\hline Stage I & 21 & 11 & 16 \\
\hline Stage II & 22 & 16 & 20 \\
\hline Stage III & 57 & 68 & 61 \\
\hline \multicolumn{4}{|l|}{ IgG type $(\%)^{b}$} \\
\hline $\operatorname{IgG}$ & 55 & Not asked & Not asked \\
\hline $\operatorname{Ig} \mathrm{A}$ & 25 & & \\
\hline $\operatorname{IgM}$ & 3 & & \\
\hline $\operatorname{IgD}$ & 0 & & \\
\hline \multicolumn{4}{|c|}{ Cytogenetic evaluation (FISH, aberrations with frequency $\geq 5 \%$ ) } \\
\hline Del 13 & 38 & 57 & 65 \\
\hline Del 17 & 12 & 16 & 10 \\
\hline $\mathrm{t}(4 ; 14)$ & 19 & 14 & 14 \\
\hline $\mathrm{t}(\mathrm{p} 16 ; \mathrm{q} 32)$ & 5 & 2 & 1 \\
\hline$\beta_{2}$-microglobulin (median in $\mathrm{mg} / \mathrm{dl}$ ) & 4.8 & 4.5 & 3.7 \\
\hline \multicolumn{4}{|l|}{ Albumin (g/dl) } \\
\hline Median & 4.4 & 4.3 & 28.0 \\
\hline Mean & 15.7 & 15.1 & 22.9 \\
\hline First line therapy $(n)$ & 429 & 242 & 335 \\
\hline \multicolumn{4}{|l|}{ Concomitant disease $^{c}(n)$} \\
\hline Renal insufficiency & 20 & 17 & 17 \\
\hline Arterial hypertension & 35 & 41 & 30 \\
\hline Coronary heart disease & 20 & 16 & 13 \\
\hline Arrhythmia & 11 & 7 & 8 \\
\hline Cardiac insufficiency & 16 & 13 & 12 \\
\hline Diabetes mellitus & 15 & 17 & 10 \\
\hline Psychiatric diagnosis (including depression) & 6 & 7 & 5 \\
\hline
\end{tabular}

${ }^{\mathrm{a}} \mathrm{As}$ not all information regarding characteristics was available, percentages may not total $100 \%$; ${ }^{\mathrm{b}} 10 \%$ unknown or other type; ${ }^{\mathrm{c}}$ occurring $\geq 5 \%$ of patients. FISH, fluorescence in situ hybridisation.

Treatment within clinical trials. Overall, $5 \%$ of patients received first-line treatment within clinical studies. University hospitals were leading with $11 \%$ of patients enrolled into clinical studies, while non-university hospitals and office-based haematologists recruited $4 \%$ of their first-line patients for clinical trials.

A total of $33 \%$ (155 of 475) of reported patients were considered eligible for ASCT following high-dose chemotherapy (Fig. 3A). Only $47 \%$ of this eligible population (representing 19\% of the survey population) actually received ASCT as part of the first-line treatment. Another $8 \%$ of patients received ASCT procedure in relapse, leading to total of $27 \%$ of patients treated with ASCT. Only a minority of ASCT-candidates received tandem ASCT (2\% of total patients, $6 \%$ of ASCT candidates). There was no obvious change in the ASCT treatment pattern; in the previous survey, a similar percentage of patients was treated with this approach (9).

First-line treatment. The median age at the time of the front-line treatment decision was 69 years in the current survey, and $42 \%$ of patients were aged $>70$ years. A minority of patients $>65$ years of age were considered for ASCT (66-70 years, $29 \%$; $>70$ years, $5 \%$ ). As expected, age was the most significant factor $(\mathrm{P}<0.001)$ influencing the decision for ASCT in the front-line setting (Fig. 3B). The decision matrix for patients above and below 69 years of age was investigated using a univariate logistical regression analysis. For elderly patients (aged $>69$ years), more advanced Durie-Salmon stage, presence of CRAB criteria (3), and presence of bone lesions were identified as factors that influenced the decision in favour of ASCT (Fig. 4A). Similarly, in the younger patient 


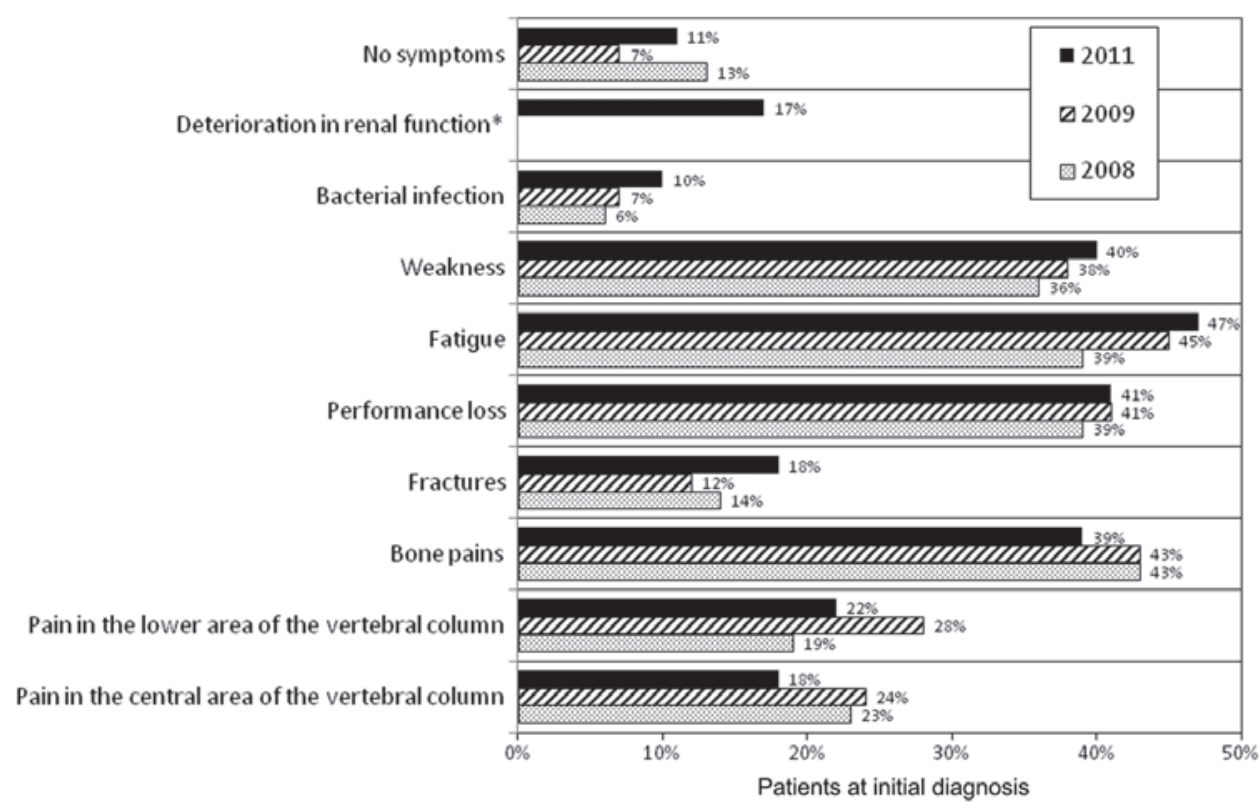

Figure 1. Leading symptoms at initial diagnosis. The percentage of patients that developed the respective symptoms is shown (only if $\geq 5 \%$ of patients). *Only included in the 2011 questionnaire.
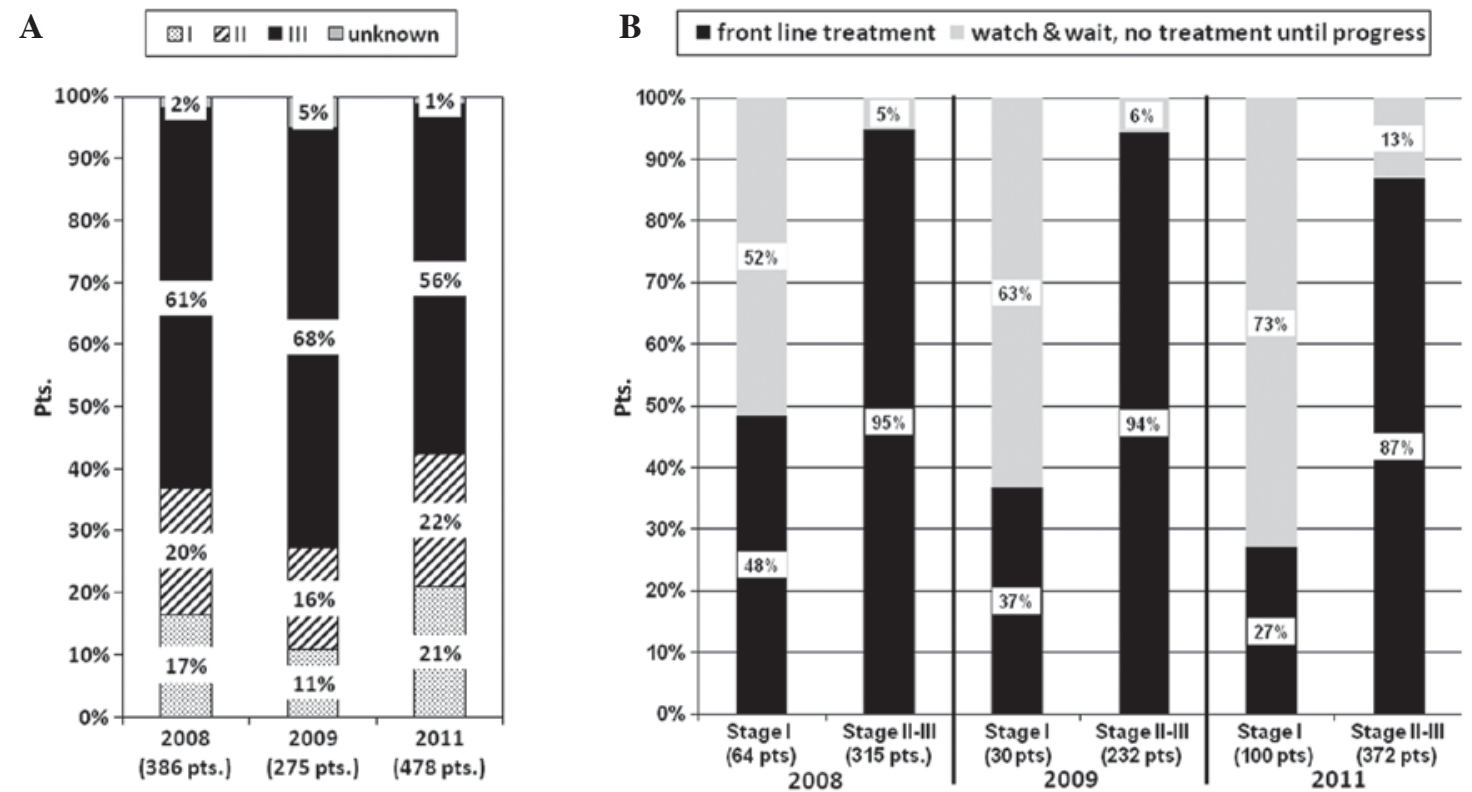

Figure 2. Distribution of patients with regard to (A) Durie-Salmon stage at initial diagnosis and (B) according to the stage of disease. Pts., patients.
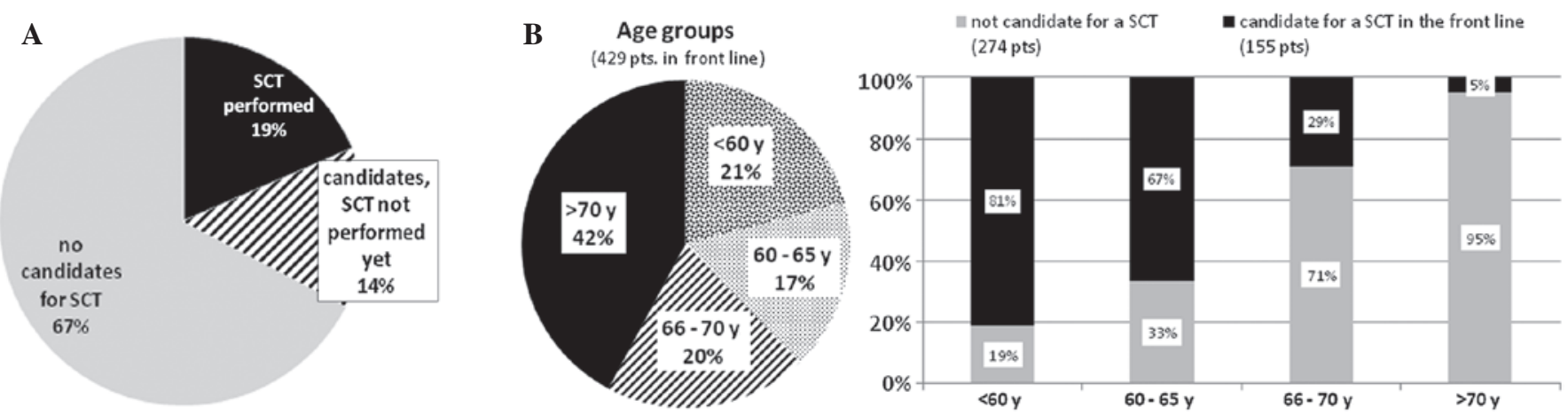

Figure 3. Transplantation-related treatment decisions for patients in first-line treatment. (A) Percentage of patients that underwent autologous blood SCT. (B) Percentage of patients treated by SCT in accordance with age groups. SCT, stem cell transplant; y, years. 


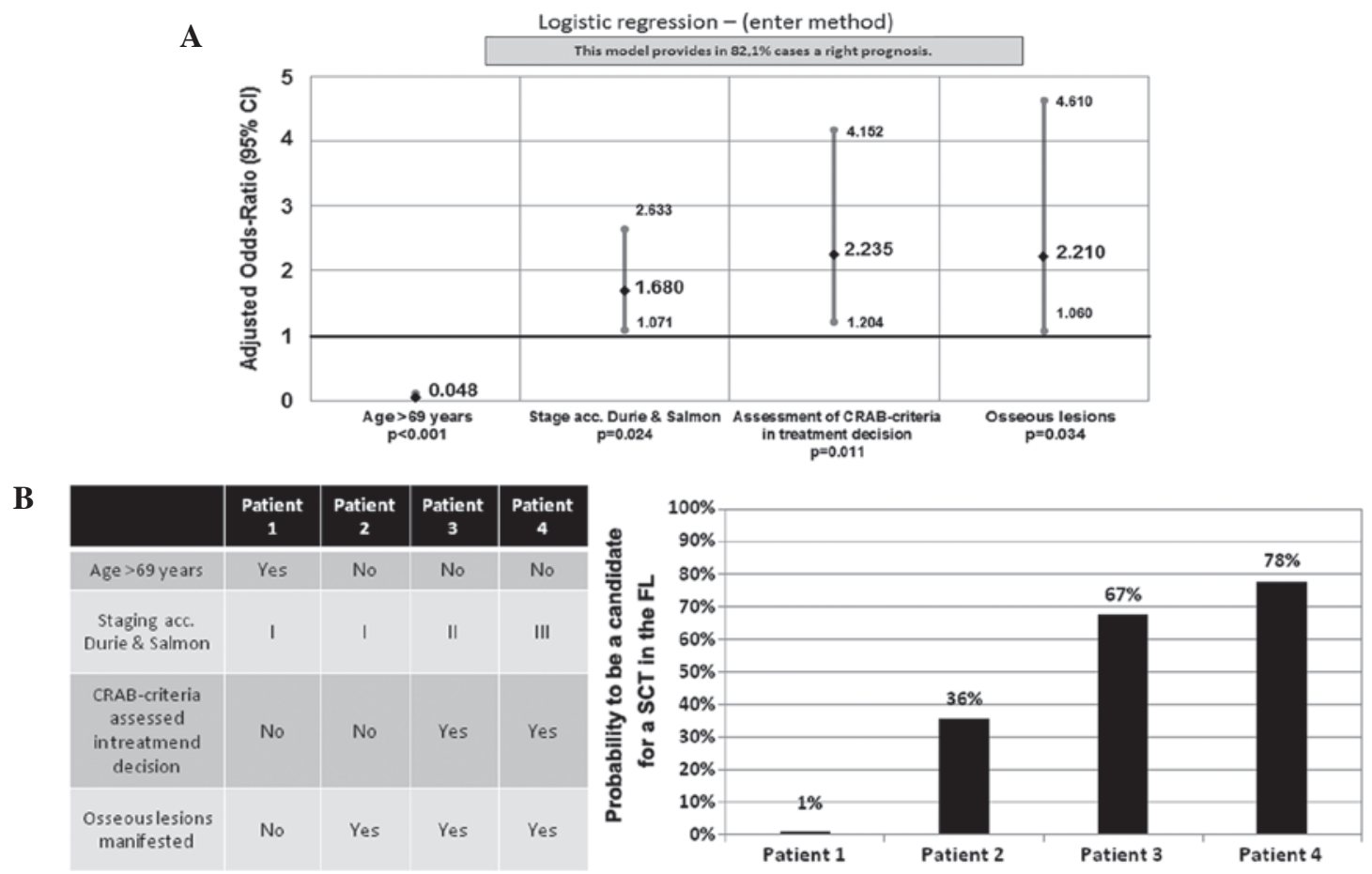

Figure 4. Patient-related factors that influenced the decision for autologous blood SCT. (A) Evaluation of the selection criteria for autologous blood SCT. (B) Prognostic models for autologous blood SCT in the FL using the following logistic regression analysis: $L$ (Logit) $=-1.386+$ ('age $>69$ ' $\mathrm{x}-3.041$ ) + ('stage DS' $x$ 0.519) + ('CRAB criteria' x 0.804) + ('manifestation of osseous lesions' x 0.793). SCT, stem cell transplant; CI, confidence interval; FL, first-line.

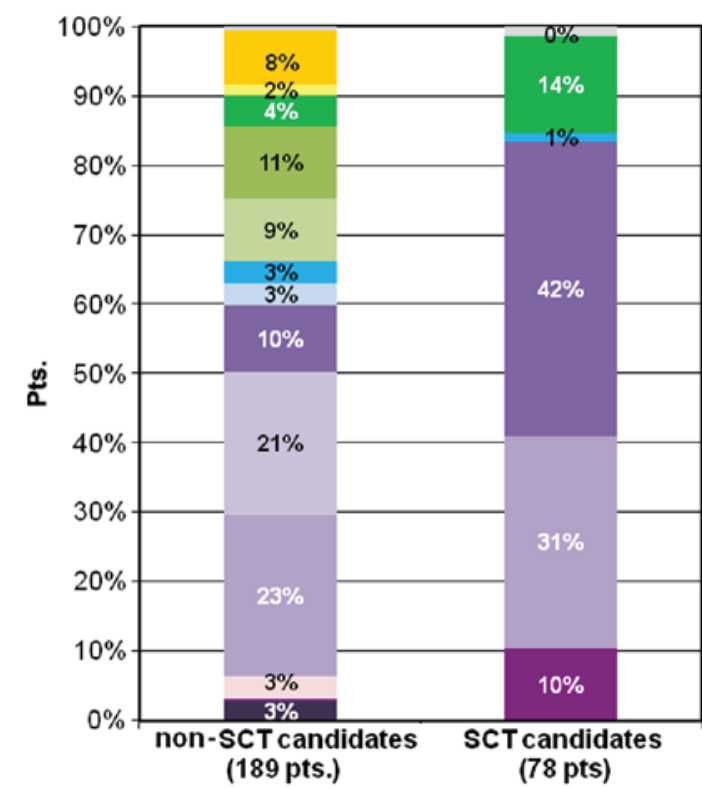

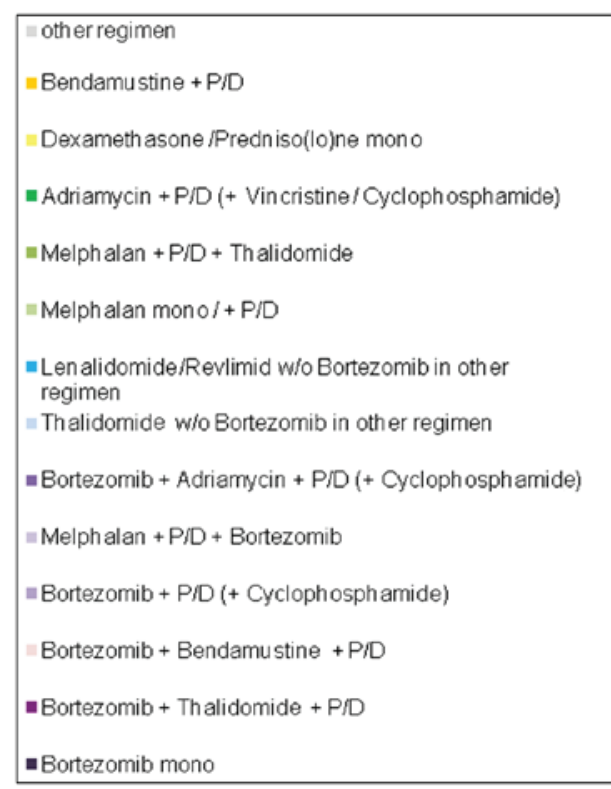

Figure 5. Comparison of first-line induction regimen for patients treated with an 'intensified' concept (including autologous blood SCT) compared to patients with a 'non-intensified' concept (no autologous blood SCT). SCT, stem cell transplant; P/D, prednisone or dexamethasone; pts., patients; w/o, without.

population (aged $<69$ years), more severe disease (defined by $C R A B$ criteria) $(\mathrm{P}=0.005)$ or diagnosis of bone lesions $(\mathrm{P}<0.001)$ influenced the decision in favour of conducting ASCT (Fig. 4B).

The decision regarding ASCT as a possible treatment option influenced the choice of chemotherapy regimen for tumour mass reduction (Fig. 5). Nevertheless, $\sim 60 \%$ of patients considered not to be an ASCT candidate received a bortezomib regimen. Despite the great variety, bortezomib-containing regimens were most frequently used in the non-intensive group (those not treated by high-dose melphalan and ASCT). Bortezomib in combination with chemotherapy was administered in 60\% of patients (Fig. 5); chemotherapy combination partners were cyclophosphamide, doxorubicin (Adriamycin), melphalan and bendamustine. Bortezomib single-agent therapy was only used for $3 \%$ of the patients. Therapy with IMiDs or IMiD-combination therapies were applied in $18 \%$ of patients that were not planned for ASCT. Only a minority (24\%) of 


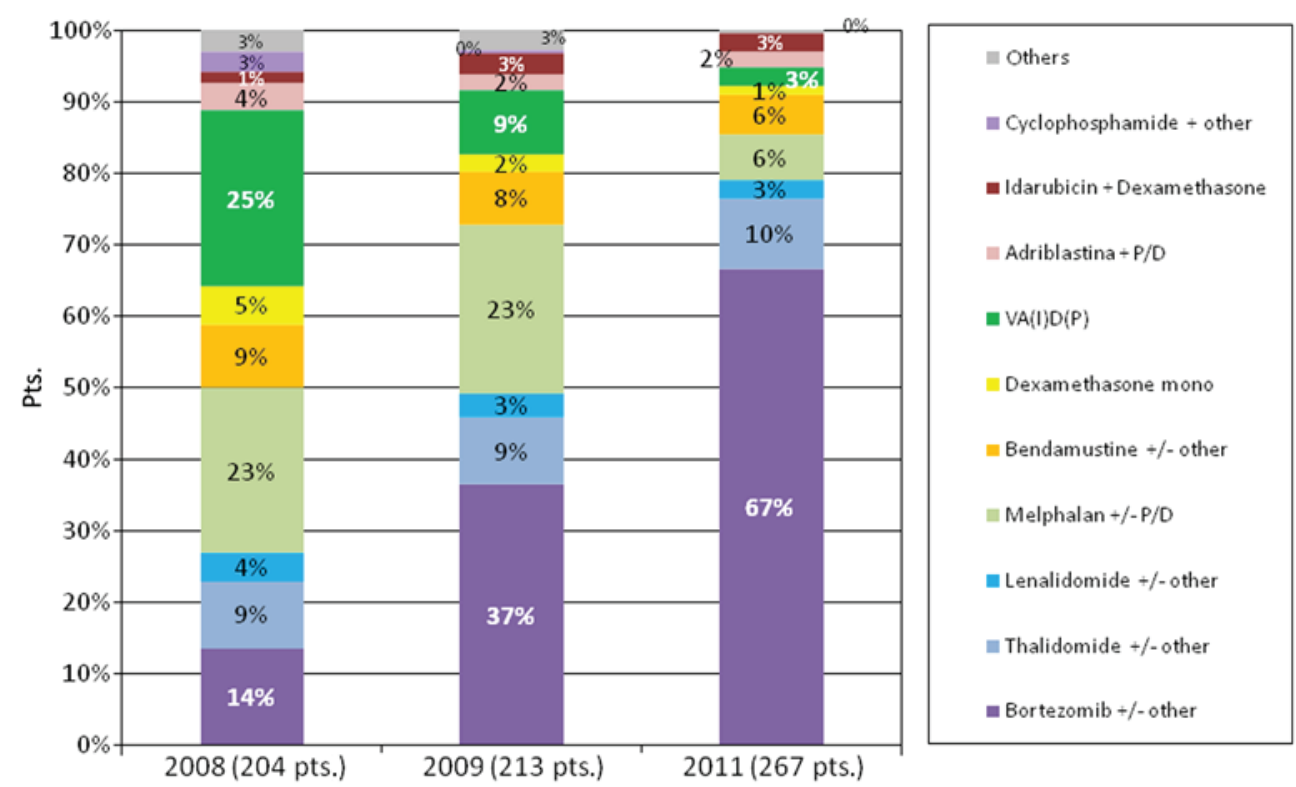

Figure 6. Shift in the front-line treatment approach between 2008 and 2011. Pts., patients; P/D, prednisone or dexamethasone; VA(I)D(P), vincristine, Adriamycin or idarubicin, dexamethasone or prednisone.

patients received a bortezomib/IMiD-free single-agent or chemo/steroid combination therapy. Melphalan (9\%) or bendamustine $(8 \%)$ were the most commonly used chemotherapies (as single agents) used in combination with corticosteroids in the current survey. Only a small percentage of patients were treated with Adriamycin-containing combinations (4\%) or steroid monotherapy (1\%) (Fig. 5).

The vast majority (83\%) of patients who were scheduled for ASCT were treated in the induction phase with bortezomib-based combination therapies (Fig. 5). The most frequently used bortezomib containing regimens were bortezomib+Adriamycin+dexamethasone (42\%), bortezomib+cyclophosphamide+dexamethasone (31\%) and bortezomib+thalidomide+prednisolone (10\%).

Among patients treated with ASCT, consolidation therapy was performed in $2 \%$ and maintenance therapy was performed in $24 \%$ of patients. Maintenance therapy was conducted within a clinical study in 38\%. The following medications were used for maintenance therapy with decreasing frequency: Thalidomide, lenalidomide and bortezomib. A small percentage $(5 \%)$ of patients received additional prednisone; however, the majority of patients were treated with a single-agent approach in the maintenance therapy period.

There was a remarkable shift in the treatment of first-line patients during 2008-2011. The change in the first-line treatment strategy over time is outlined in Fig. 6. As described, bortezomib combinations have predominantly substituted all traditional novel-agent-free chemotherapy regimens, such as melphalan+prednisone and vincristin+Adriamycin+dexamet hasone. Currently, the IMiDs thalidomide and lenalidomide serve a remarkably constant, but substantially smaller, role in first-line treatment.

Impact of concomitant disease, particularly renal impairment, on treatment decisions. In the current survey, $16 \%$ of patients in the first-line setting suffered from renal impairment (Fig. 7A). For a majority of patients (63\%), no renal impairment and only one concomitant disease was reported, and multimorbidity without renal impairment was reported in $21 \%$ of patients. The analysis of treatment patterns in these three patient subgroups revealed a significant $(\mathrm{P}<0.001)$ impact of the morbidity on the treatment pattern. While $27 \%$ of patients with $\leq 1$ concomitant disease were treated with ASCT, $<10 \%$ of patients with renal impairment or with $\geq 2$ concomitant diseases received ASCT (Fig. 7B).

As indicated in Fig. 8, the agents used for the treatment of patients with renal impairment are similar to the agents used for first-line treatment in patients with normal renal function $(\mathrm{P}=0.097)$.

Therapy in first relapse. Fig. 9 indicates the treatment approach selected for first-relapse. Since bortezomib was approved (in 2008) and adopted rapidly in the first-line treatment setting, the number of patients treated with bortezomib in the second-line setting decreased. A substantial increase in IMiD-containing, and particularly lenalidomide-containing, regimens was observed. A switch from bortezomib in the first-line to lenalidomide was performed, in particular, for patients in whom the response duration to the bortezomib first-line regimen was $<6$ months. Overall, therapy with lenalidomide and, to a smaller extent, thalidomide has become an important aspect of treatment for relapsed disease. Melphalan+corticosteroid regimens have almost disappeared from second-line treatment.

Supportive therapy. As expected, $>90 \%$ of patients received at least some form of supportive care with first-line treatment. As indicated in Fig. 10, treatment with bisphosphonates was the leading supportive care therapy. There appears to be a declining necessity for additional supportive therapy since 2008 (Fig. 10); in particular, there is a trend for reduced use of erythropoietin-stimulating agents (ESAs), granulocyte colony-stimulating factor (G-CSF) and substitution of red blood cells and platelets, respectively. ESA use was reduced by $90 \%$, while G-CSF, red blood cell and platelet transfusions were reduced by $50 \%$. 


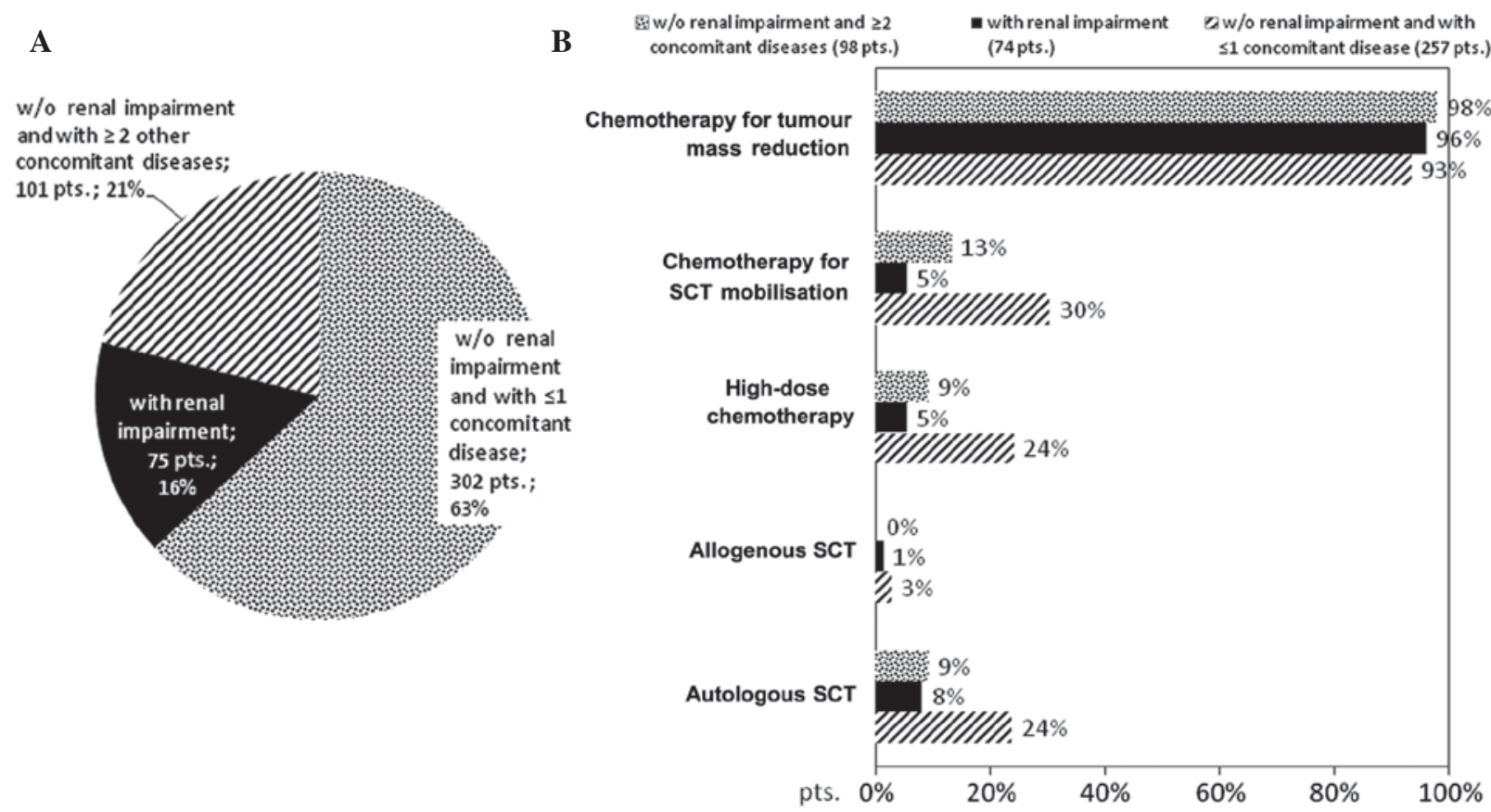

Figure 7. Influence of renal function on front-line treatment. (A) Comparison of the front-line treatment and association with comorbidity (no significant difference). (B) Spectrum of comorbidity focussed on renal impairment. Pts., patients; w/o, without; SCT, stem cell transplant.

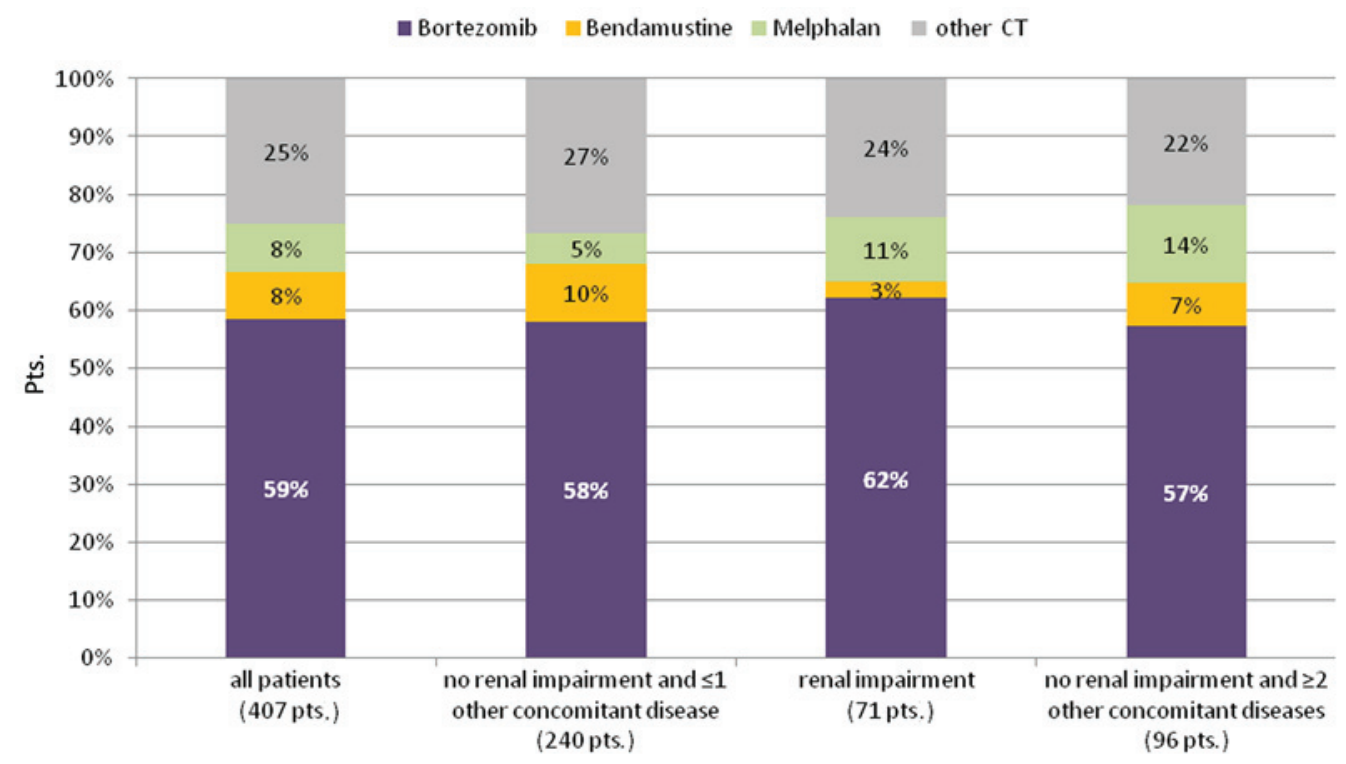

Figure 8. Selection of the first-line CT in relation to renal impairment and other comorbidities (no significant difference, $\mathrm{P}=0.097$ ). Pts., patients; CT, chemotherapy.

\section{Discussion}

The current analysis of data regarding multiple myeloma patients provides information on real-world treatment and diagnostic decisions to clarify the information on which regimen and treatment pathways are currently favoured by clinicians and on which regimen could be the basis for the future development of myeloma care.

The patient population was very comparable between the surveys with regard to participating institutions, covering office-based haemato-oncologists, non-academic hospitals and university hospitals. Age range, and the percentages of patients in each Durie-Salmon stage and asymptomatic patients, were comparable across the participating institutions. There are four major findings from the current study:

Firstly, there was a clear increase of cytogenetic assessment, from $22 \%$ of patients analysed in the 2008 survey, to $53 \%$ of patients in the current survey. The cytogenetic risk profile as well as the $\beta_{2}-\mathrm{MG}$ level can be demonstrated to be integrated into the treatment decisions (12). As age and comorbidity are the most important factors for selection of treatment regimen, elderly patients (aged $>69$ years) with reduced performance status had the lowest frequency for cytogenetic testing. Thus, treating physicians demonstrated great consideration towards resource utilisation in the diagnostic setting. 


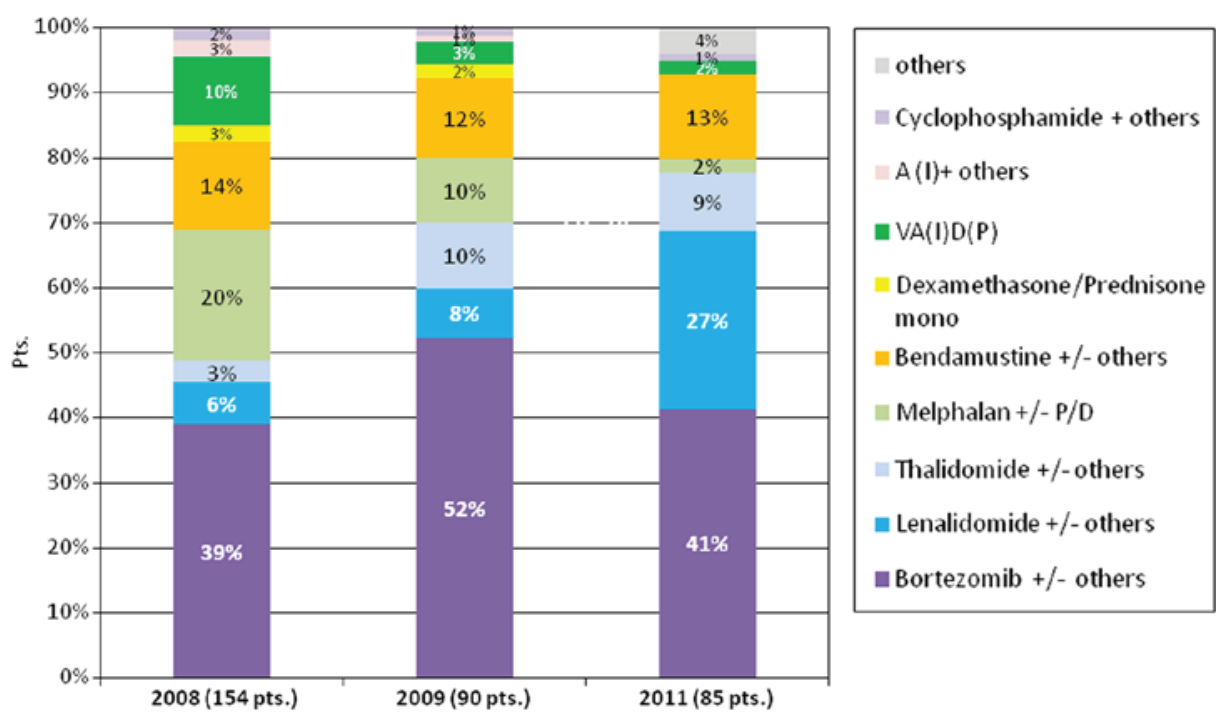

Figure 9. Shift in therapeutic regimen in the second line between 2008 and 2011. Pts., patients; A(I), Adriamycin or idarubicin; VA(I)D(P), vincristine, Adriamycin or idarubicin, dexamethasone or prednisone; $\mathrm{P} / \mathrm{D}$, prednisone or dexamethasone.

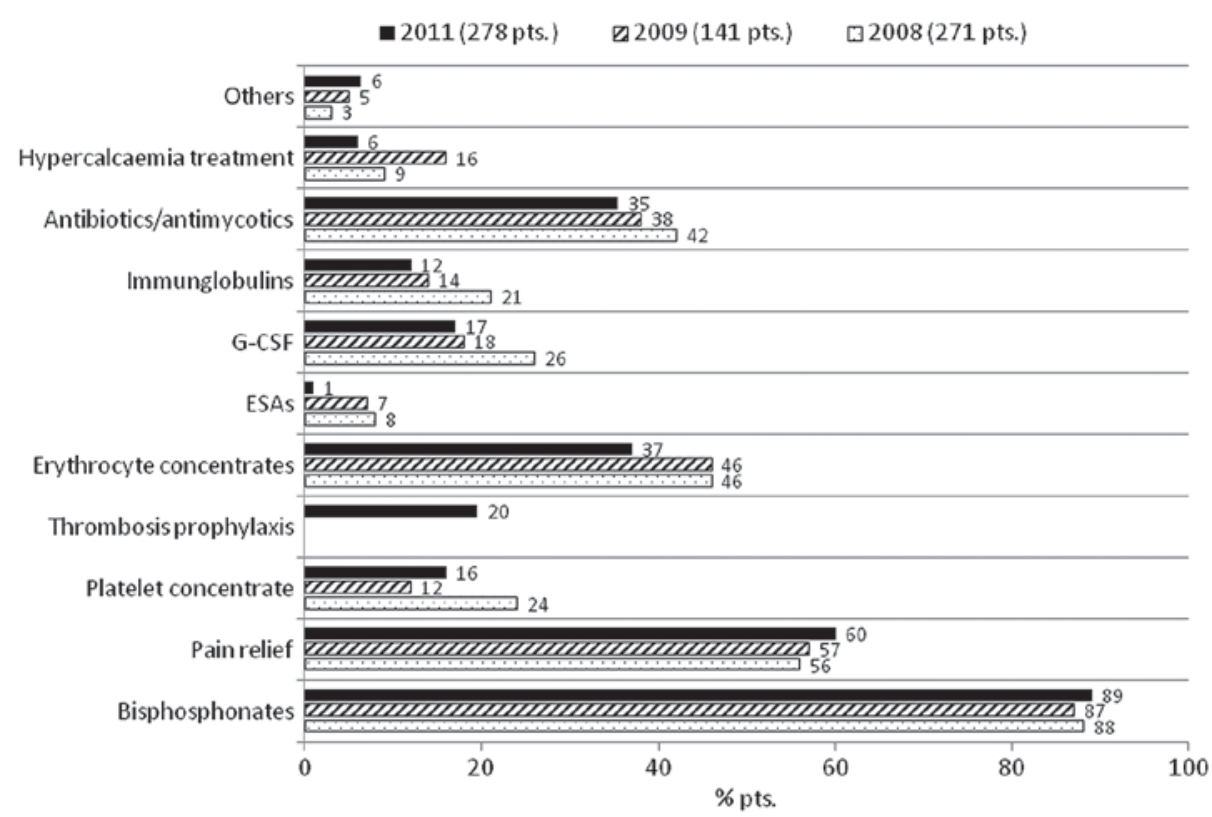

Figure 10. Shift in supportive care in first-line treatment of multiple myeloma patients. Type of supportive therapy as well as year of assessments are indicated and compared. Pts., patients; G-CSF, granulocyte colony-stimulating factor; ESAs, erythropoiesis-stimulating agents.

Secondly, bortezomib-based regimens have become the standard of care for first-line therapy for German myeloma patients, regardless of whether the patients are eligible for ASCT, or are treated in a university hospital or community-based centre. ASCT for eligible patients is still recognised as an essential component of therapy. Although novel agents substantially improved the outcome of first-line therapy, the use of ASCT has not declined over the past years. Importantly, despite data showing that overall survival does not differ comparing upfront and delayed ASCT (13), the following arguments still favour ASCT for eligible patients: The long treatment-free interval, the high quality of life following ASCT, and the possibility for long-term disease-free survival beyond 14 years (14). In consequence, only $8 \%$ of patients in the current survey received a delayed ASCT procedure $(13,15)$. The concept of upfront ASCT was recently validated by Palumbo et al (16) in a randomised phase III study that demonstrated the advantage of front-line ASCT compared to therapy with a novel agent-containing regimen. The present survey also indicates that the decision for ASCT is based on various factors, including age and myeloma-induced organ dysfunction; this is unexpected, as most guidelines indicate the decision for ASCT as an age-dependent decision, but one independent of myeloma-related disease (17).

Maintenance treatment was used in $24 \%$ of patients subsequent to ASCT, and in 38\% of patients within a clinical study. Several clinical studies suggest that maintenance therapy improves progression-free survival and overall survival times, despite some remaining concern regarding the rate of secondary malignancy in patients undergoing long-term IMiD 
treatment (18-21). Overall, we conclude that it is becoming a broader consensus in the community to focus on improving the response quality and using maintenance treatment as part of this strategy.

The third important finding from the current study is that the use/switch of novel agents for relapsed disease is in line with international standards (2). For patients relapsing within 6 months after primary therapy, lenalidomide is the most commonly applied agent; whereas, in patients with prolonged remission after primary therapy, re-treatment with bortezomib is a considered a valid option. Furthermore, thalidomide appeared to be used more frequently in community-based offices as third-line treatment as compared with university hospitals. This finding may reflect cost considerations, taking into account the expensiveness of lenalidomide. Although it has been demonstrated that autologous re-transplantation is suitable for patients with sustained remission following initial ASCT (22), the current survey indicates that this has only a marginal relevance in general practice. The same holds true for allogeneic transplantation in relapsed disease.

The fourth major finding was that the general need for supportive care in addition to bisphosphonates declined in the last years. We hypothesise that the improved treatment outcomes reduced the requirement for supportive care such as pain relief and haematopoietic growth factors. It is also likely that the use of IMiDs, which have an erythrocyte-stimulating side effect, contributed to this. As economical discussions will go on, it is important to integrate assessment of quality of life and individual work performance into phase III and IIIb/IV clinical studies (8).

With the aforementioned measures, care was taken to counteract the potential limitations of the data collection approach in the current study. The consistency of some of the key parameters, such as patient characteristics, across the treatment survey indicates that the presented data are robust. In addition, response data was not presented in this data analysis, as this information would be largely unreliable and a topic for prospective clinical studies.

In conclusion, the current study demonstrates a significant modification of treatment patterns for elderly and younger multiple myeloma patients based on the findings of international studies, indicating a rapid adoption of clinical study results into clinical practice. The survey also indicates that four-drug combinations in first-line treatment are not well established.

\section{References}

1. Merz M, Neben K, Raab MS, Sauer S, Egerer G, Hundemer M, Hose D, Kunz C, Heiß C, Ho AD, et al: Hillengass Autologous stem cell transplantation for elderly patients with newly diagnosed multiple myeloma in the era of novel agents. Ann Oncol 25: 189-195, 2014.

2. Ludwig H, Avet-Loiseau H, Bladé J, Boccadoro M, Cavenagh J, Cavo M, Davies F, de la Rubia J, Delimpasi S, Dimopoulos M, et al: European perspective on multiple myeloma treatment strategies: Update following recent congresses. Oncologist 17: 592-606, 2012.

3. Ludwig H, Miguel JS, Dimopoulos MA, Palumbo A, Garcia SanzR, Powles R, Lentzsch S, Ming Chen W, Hou J, Jurczyszyn A, et al: Durie International myeloma working group recommendations for global myeloma care. Leukemia 28: 981-992, 2014.

4. Pulte D, Gondos A and Brenner H: Improvement in survival of older adults with multiple myeloma: Results of an updated period analysis of SEER data. Oncologist 16: 1600-1603, 2011.
5. Brenner H, Gondos A and Pulte D: Recent major improvement in long-term survival of younger patients with multiple myeloma. Blood 111: 2521-2526, 2008.

6. Klein B, Seckinger A, Moehler T and Hose D: Molecular pathogenesis of multiple myeloma: Chromosomal aberrations, changes in gene expression, cytokine networks, and the bone marrow microenvironment. Recent Results Cancer Res 183: 39-86, 2011.

7. Engelhardt M, Terpos E, Kleber M, Gay F, Wäsch R, Morgan G, Cavo M, van de Donk N, Beilhack A, Bruno B, et al: European myeloma network recommendations on the evaluation and treatment of newly diagnosed patients with multiple myeloma. Haematologica 99: 232-242, 2014.

8. Gaultney JG, Redekop WK, Sonneveld P and Uyl-de Groot CA Novel anticancer agents for multiple myeloma: A review of the evidence for their therapeutic and economic value. Expert Rev Anticancer Ther 12: 839-854, 2012.

9. Knauf W, Kellermann L, Poenisch W, Einsele H, Straka C, Frohn C and Goldschmidt $\mathrm{H}$ : How to treat multiple myeloma - a representative multicentre treatment survey. Onkologie 33: 604-610, 2010.

10. Durie BG and Salmon SE: A clinical staging system for multiple myeloma. Correlation of measured myeloma cell mass with presenting clinical features, response to treatment, and survival. Cancer 36: 842-854, 1975.

11. Greipp PR, San Miguel J, Durie BGM, Crowley JJ, Barlogie B, Blade J, Boccadoro M, Child A, Avet-Loiseau H, Kyle RA, et al: International staging system for multiple myeloma. J Clin Oncol 23: 3412-3420, 2005.

12. Moreau P, Cavo M, Sonneveld P, RosinolL, Attal M,Pezzi A, Goldschmidt H, Lahuerta JJ, Marit G, Palumbo A, et al: Combination of international scoring system 3 , high lactate dehydrogenase and $\mathrm{t}(4 ; 14)$ and/or del (17p) identifies patients with multiple myeloma (MM) treated with front-line autologous stem-cell transplantation at high risk of early MM progression-related death. J Clin Oncol 32: 2173-2180, 2014.

13. Fermand JP, Ravaud P, Chevret S, Divine M, Leblond V, Belanger C, Macro M, Pertuiset E, Dreyfus F, Mariette X, et al: High-dose therapy and autologous peripheral blood stem cell transplantation in multiple myeloma: Up-front or rescue treatment? Results of a multicenter sequential randomized clinical trial. Blood 92: 3131-3136, 1998.

14. van Rhee F Giralt S and Barlogie B: The future of autologous stem cell transplantation in myeloma. Blood 124: 328-333, 2014.

15. Fermand JP, Katsahian S, Divine M, Leblond V, Dreyfus F, Macro M, Arnulf B, Royer B, Mariette X, Pertuiset E, et al: High-dose therapy and autologous blood stem-cell transplantation compared with conventional treatment in myeloma patients aged 55 to 65 years: Long-term results of a randomized control trial from the Group Myelome-Autogreffe. J Clin Oncol 23: 9227-9233, 2005.

16. Palumbo A, Cavallo F, Gay F, Di Raimondo F, Ben Yehuda D, Petrucci MT, Pezzatti S, Caravita T, Cerrato C, Ribakovsky E, et al: Autologous transplantation and maintenance therapy in multiple myeloma. N Engl J Med 371: 895-905, 2014.

17. Ludwig H, Beksac M, Bladé J, Boccadoro M, Cavenagh J, Cavo M, Dimopoulos M, Drach J, Einsele H, Facon T, et al: Current multiple myeloma treatment strategies with novel agents: A European perspective. Oncologist 15: 6-25, 2010.

18. Sonneveld P, Schmidt-Wolf IG, van der Holt B, El Jarari L, Bertsch U, Salwender H, Zweegman S, Vellenga E, Broyl A, Blau IW, et al: Bortezomib induction and maintenance treatment in patients with newly diagnosed multiple myeloma: Results of the randomized phase III HOVON-65/ GMMG-HD4 trial. J Clin Oncol 30: 2946-2955, 2012.

19. Palumbo A, Hajek R, Delforge M, Kropff M, Petrucci MT, Catalano J, Gisslinger H, Wiktor-Jędrzejczak W, Zodelava M, Weisel K, et al: Continuous lenalidomide treatment for newly diagnosed multiple myeloma. N Engl J Med 366: 1759-1769, 2012.

20. McCarthy PL, Owzar K, Hofmeister CC, Hurd DD, Hassoun H, Richardson PG, Giralt S, Stadtmauer EA, Weisdorf DJ, Vij R, et al: Lenalidomide after stem-cell transplantation for multiple myeloma. N Engl J Med 366: 1770-1781, 2012.

21. Palumbo A, Bringhen S, Kumar SK, Lupparelli G, Usmani S, Waage A, Larocca A, van der Holt B, Musto P, Offidani M, et al: Second primary malignancies with lenalidomide therapy for newly diagnosed myeloma: A meta-analysis of individual patient data. Lancet Oncol 15: 333-342, 2014.

22. Sellner L, Heiss C, Benner A, Raab MS, Hillengass J, Hose D, Lehners N, Egerer G, Ho AD, Goldschmidt $\mathrm{H}$ and Neben K: Autologous retransplantation for patients with recurrent multiple myeloma: A single-center experience with 200 patients. Cancer 119: 2438-2446, 2013. 